Instructions for authors, subscriptions and further details:

http://rimcis.hipatiapress.com

\title{
Aprender A Ser Docente Sin Estar En Las Aulas: La COVID-19 Como Amenaza Al Desarrollo Profesional Del Futuro Profesorado
}

Gustavo González-Calvo ${ }^{1}$, Raúl A. Barba-Martín ${ }^{1}$, Daniel Bores-García², Vanesa Gallego-Lema ${ }^{3}$

1) University of Valladolid

2) University Rey Juan Carlos

3) University of the Basque Country

Date of publication: article first published online June, 29 2020; Issue published July, 302020.

Edition period: July 2020 - November 2020

To cite this article: González-Calvo, G., Barba-Martín, R.A., Bores-García, D. \& Gallego-Lema, V. (2020). Aprendiendo a Ser Docente Sin Estar en las Aulas. La COVID-19 Como Amenaza al Desarrollo Profesional del Futuro Profesorado. International and Multidisciplinary Journal of Social Sciences, 2(9), 152-177. doi : 10.17583/rimcis.2020.5783

To link this article: http://doi.org/10.17583/rimcis.2020.5783

\section{PLEASE SCROLL DOWN FOR ARTICLE}

The terms and conditions of use are related to the Open Journal System and to Creative Commons Attribution License (CC-BY). 


\title{
Learning To Be A Teacher Without Being In The Classroom: COVID-19 As A Threat To The Professional Development Of Future Teachers
}

\author{
Gustavo González-Calvo \\ University of Valladolid \\ Raúl A. Barba-Martín \\ University of Valladolid
}

Daniel Bores-García

University Rey Juan Carlos

Vanesa Gallego-Lema

University of the Basque Country

\section{Abstract}

Teachers in initial training who attend the Practicum find, in this period, a meaning for the teaching profession, awakening a reflective and critical attitude about what and how they do things, and therefore, shaping their teaching identity. However, in recent months, the training situation of future teachers has taken a turn due to the health crisis caused by the COVID-19 pandemic. The study carried out focused on inquiring, through a narrative research, how university students have perceived the period of practices they carried out through virtual teaching, as a consequence of the current health crisis. Among other results, the evidence showed that students developed insecurity feelings, melancholy and uncertainty in light of the new learning processes. On the other hand, by losing on-site classroom attendance and with it the essence of the practices, the training potential of the Practicum and the teaching professional identity of the students have been affected during these months, consequences that could affect their future professional practices.

Keywords: teacher identity, COVID-19, higher education, preservice teacher education 
Sciences Vol. 9 No.2 July 2020 pp. 152-177

\section{Aprender A Ser Docente Sin Estar En Las Aulas: La COVID-19 Como Amenaza Al Desarrollo Profesional Del Futuro Profesorado}

Gustavo González-Calvo

Universidad de Valladolid

Raúl A. Barba-Martín

Universidad de Valladolid
Daniel Bores-García

Universidad Rey Juan Carlos

Vanesa Gallego-Lema

Universidad del País Vasco

\section{Resumen}

Las y los docentes en formación inicial que acuden al Prácticum encuentran en este periodo un sentido a la profesión docente, despertando en ellos una actitud reflexiva y crítica sobre qué hacen y cómo lo hacen, y por tanto, conformando su identidad docente. Sin embargo, en los últimos meses la situación formativa de las y los futuros educadores ha dado un giro debido a la crisis sanitaria originada por la pandemia de la COVID-19. El estudio llevado a cabo se centró en indagar, mediante una investigación narrativa, cómo las y los estudiantes universitarios han percibido el periodo de prácticas que realizaron por medio de una enseñanza virtual, como consecuencia de la actual crisis sanitaria. Entre otros resultados, las evidencias mostraron que el alumnado desarrolló sentimientos de inseguridad, melancolía e incertidumbre antes los nuevos procesos de aprendizaje. Por otro lado, al perder la presencialidad en las aulas y con ello la esencia de las prácticas, el potencial formativo del Prácticum y la identidad profesional docente del estudiantado se han visto alteradas durante estos meses, consecuencias que podrían afectar a sus futuras prácticas profesionales.

Palabras clave: identidad docente, COVID-19, educación superior, formación inicial del profesorado 
L

a etapa de formación inicial es el periodo en la que las y los futuros educadores comienzan a analizar, examinar, observar y reflexionar sobre lo que hace y el modo en que lo hace; es el vehículo que les permite ir descubriendo su propia identidad de manera crítica y reflexiva (Atkinson, 2004; Graham \& Phelps, 2003). Uno de los rasgos fundamentales para descubrir la identidad pedagógica de las y los futuros docentes radica en la esencia interpersonal de la profesión (González-Calvo \& Fernández-Balboa, 2018), en el modo en que el profesorado en formación interactúa con el alumnado y el resto de la comunidad educativa. No obstante, la profesión ha cambiado radicalmente en los últimos meses debido a la crisis sanitaria de la COVID-19. España, como otros tantos países, ha optado por decretar un estado de alarma y, con ello, el cierre de escuelas y universidades. Se ha evidenciado en este tiempo que profesiones como la enseñanza han cambiado de forma presencial a forma virtual en cuestión de horas, una situación que podría ser problemática para las y los futuros docentes que se encuentran realizando sus prácticas en los centros escolares y se han visto privados de la socialización de la profesión dentro de las aulas.

Partiendo de la premisa de que las identidades docentes pueden ser interpretadas desde un enfoque narrativo (Loughran, 2005; RuohotieLyhty, 2013; Søreide, 2006; Watson, 2006), este estudio se apoya en la investigación narrativa como técnica de reflexión y toma de conciencia de las creencias y planteamientos que, sobre la educación en general y sobre la educación virtual en tiempos de pandemia, tiene un grupo de estudiantes de último curso del Grado en Educación Primaria que se encontraban realizando sus prácticas. Comprender y analizar las narrativas de las y los participantes permite ser conscientes de cómo sus experiencias, expectativas y temores en tiempos de crisis marcan su modo de entender y orientar la profesión. Así, el objetivo de este artículo es explorar la manera en que el alumnado en formación inicial experimenta su periodo de prácticas de manera virtual como consecuencia de la actual crisis sanitaria. De manera más específica, profundizamos en cómo la realización de las prácticas "sin presencialidad" condiciona su práctica pedagógica y su manera de entender la profesión. Dada la emergente y reciente situación sanitaria 
originada por la pandemia, han surgido ya estudios relacionados con los cambios producidos en la docencia (Diez-Gutiérrez \& GajardoEspinoza, 2020; Trujillo et al., 2020). Sin embargo, este trabajo es pionero en investigar cómo los nuevos procesos formativos virtuales afectan a la identidad docente en la formación inicial de profesorado.

\section{Formación Inicial Docente y Desarrollo De La Identidad Profesional}

En varios estudios se ha demostrado que las creencias y experiencias previas actúan a modo de filtro respecto a los conocimientos que se imparten en la universidad (Atkinson, 2004; Imbernón Muñoz, 2007; Orland-Barak \& Maskit, 2011). También sabemos que la historia personal presenta intersecciones con las competencias profesionales (Day et al., 2005; Graham \& Phelps, 2003; Hong, 2010; Lopes, 2009; Rodgers \& Scott, 2008), al tiempo que la formación inicial ayuda a la transformación de lo aprendido por socialización como estudiante (Atkinson, 2004; Graham \& Phelps, 2003). Sin embargo, la educación en general y más en particular la universitaria, actúa dejando al margen las experiencias y creencias previas como si la enseñanza que promueve estuviera aislada en sí misma. En este punto, entran en juego las narrativas y los procesos reflexivos como oportunidad para (re)interpretar y (re)imaginar las historias personales y profesionales, provocando un desplazamiento de la mirada y una (re)significación de la identidad profesional docente (e.g. González-Calvo \& AriasCarballal, 2017; González-Calvo \& Fernández-Balboa, 2018).

La identidad docente, en cualquier caso, no es una cuestión de pertenencia, sino más bien un conjunto de necesidades, valores, experiencias, sentimientos y habilidades que se forman a lo largo de la experiencia personal y profesional de cada cual, creando dicho sentido de identidad (Korthagen, 2004, 2010). A lo largo de todo este proceso es esencial la formación inicial, pues actúa como referente en la preparación de educadoras y educadores capaces de evolucionar, de aprender de la experiencia, de reflexionar sobre su práctica y aprender de todo ello (Perrenoud, 2010). Por este motivo, estamos convencidos de que el periodo de prácticas -el denominado Prácticum en los 
programas universitarios españoles- es un tiempo ideal para encontrar sentido a la profesión docente. Así, en este periodo se habrá de procurar despertar una práctica reflexiva entre las y los estudiantes que se preparan para desempeñar, en un futuro próximo, su práctica pedagógica, procurando con ello organizar, desarrollar y enriquecer la que será su docencia y seña de identidad como educadora y educador (Pence \& Macgillivray, 2008). Al reflexionar sobre su propia práctica y experiencias personales, el futuro educador o educadora irá desarrollando habilidades como observar lo que sucede en el aula, analizar las razones de determinadas actuaciones y planificar nuevas y mejores propuestas didácticas, entre otras. En síntesis, estará en condiciones de pensar, de una manera sistemática, acerca de lo ocurrido, el significado de lo ocurrido y las implicaciones de esas acciones sobre el alumnado y sobre sí mismo.

Otro aspecto que también repercute al ámbito de la formación son los cambios socio-culturales, provocando en las y los estudiantes una continua adaptación y aprendizaje que afecta a su identidad docente. Actualmente, el mayor giro ha sido la aparición de la enfermedad COVID-19, que ha supuesto que en España se decrete el estado de alarma el 14 de marzo. Una de las primeras medidas tomadas ha sido el cierre de los centros educativos, que supuso la suspensión de las actividades docentes de carácter presencial para dar paso a un formato online (García-Peñalvo et al., 2020). Si previamente a esta situación, un aprendizaje virtual suponía tener en cuenta ciertas limitaciones o problemáticas, como por ejemplo aquellas de carácter tecnológico, metodológico, formativos, entre otras; con la actual readaptación urgente de las clases a un formato virtual y sin tiempo para una transformación digital, conlleva un reto para las instituciones y para las comunidades educativas (García-Peñalvo et al., 2020; Llorens-Largo \& Fernández, 2020). Por lo tanto, nos encontramos en un contexto en el que las y los futuros docentes tienen que aprender a navegar en tiempos de incertidumbre (Britzman, 2007), y en el que su identidad docente se ve afectada al construirse procesualmente de manera subjetiva y social (Bolívar \& Domingo, 2019). 


\section{Metodología}

La metodología adoptada en este estudio es la investigación narrativa, la cual profundiza en las experiencias vividas (Clanindin, 2007), poniendo el foco en el deseo de comprender los sentidos, las identidades, las experiencias humanas, partiendo de lo singular para escuchar a las y los demás (Bolívar \& Domingo, 2019). Concretamente en esta investigación, la comprensión y análisis de las narrativas de las y los participantes nos permite ser conscientes de cómo sus experiencias, expectativas y temores en tiempos de crisis marcan su modo de entender y orientar la profesión. Utilizamos el modelo de microetnografía (Le Baron, 2006), con el objeto de estudiar partes concretas de las experiencias de un grupo de personas durante un corto periodo de tiempo.

\section{Participantes}

Las y los participantes en este estudio fueron ocho estudiantes de entre 22 y 25 años de edad. Todos estaban cursando, en el momento del estudio, el último curso del Grado en Educación Primaria, especialidad en Educación Física, en una universidad pública española.

\section{Recopilación De Datos}

La recopilación de datos comenzó poco después de que las y los participantes aceptaran participar en el estudio y firmaran un formulario de consentimiento. Cada participante se sometió a una entrevista semiestructurada que duró entre 60 y 90 minutos. Para aumentar la fiabilidad del estudio (Lincoln \& Guba, 1985) y fomentar el análisis de los datos, las entrevistas se grabaron en audio y se transcribieron textualmente.

Se pidió al alumnado participante que proporcionaran narraciones que incluyeran sus percepciones y experiencias sobre: (a) qué sentimientos afloran en ellos al tener que enfrentarse a su periodo de prácticas en tiempos de pandemia; (b) qué ventajas e inconvenientes tiene la enseñanza virtual respecto de la presencial; y (c) de qué manera 
la crisis sanitaria ha cambiado su percepción hacia la enseñanza y su práctica pedagógica.

Con el fin de fomentar narraciones libres y abiertas sobre estos temas, el estudiantado participante eligió el momento de las entrevistas y se le garantizó el anonimato mediante el uso de seudónimos. Además, las entrevistas se llevaron a cabo de manera informal, en las que las y los investigadores actuaron como oyentes activos $\mathrm{e}$ instaron gentilmente a las y los participantes a generar relatos detallados relacionados con los propósitos del estudio (Smith, 2010). Debido a la situación de crisis sanitaria, las entrevistas se realizaron de manera virtual, a través del programa Teams (Microsoft).

\section{Análisis De Los Datos}

Toda la información obtenida se analizó mediante análisis de contenido temático (Libarkin \& Kurdziel, 2002) y comparación constante (Miles \& Huberman, 1994). El análisis de contenido temático se centra en la búsqueda de patrones en el texto. Se utilizó una codificación descriptiva y de patrones para analizar tanto los patrones dentro del caso como los patrones cruzados. Utilizando las transcripciones de las entrevistas, se leyeron varias veces los comentarios del alumnado participante para tener una idea general del contenido en su conjunto hasta conseguir claridad y, también, para identificar unidades y patrones de significado que más tarde servirían para generar las categorías y subcategorías, identificar citas útiles y establecer la teoría general para las conclusiones y el debate del estudio (Dale, 2000). Además, un experto en métodos cualitativos llevó a cabo exámenes por homólogos para establecer la credibilidad en la fase de análisis de los datos (Sparkes \& Smith, 2014). Inicialmente, esto condujo a la identificación de las principales categorías o temas. Luego, las y los investigadores emprendieron una segunda ronda de análisis a través de un proceso de comparación constante de los textos a fin de establecer subtemas creíbles y fiables en cada categoría (Gubrium \& Holstein, 2009). Posteriormente, se seleccionaron citas pertinentes para ilustrar estos temas y subtemas. 
Los temas emergentes que surgen del análisis de datos estaban relacionados con la situación actual a la que han de enfrentarse las y los docentes y los sentimientos que afloran, el cambio de las convicciones pedagógicas antes y durante la crisis sanitaria, cómo afrontan la enseñanza de la Educación Física de manera virtual y las consecuencias que tiene no poder cumplir como educadoras y educadores físicos en una situación real sino mediada por la tecnología.

\section{Resultados}

El análisis de los datos revela tres temas principales en relación a la práctica pedagógica de las y los futuros docentes y los efectos que la enseñanza virtual, a la que ha obligado la pandemia, tiene sobre su práctica; a saber: (a) las consecuencias que ha tenido perder el factor presencial dentro de su formación inicial; (b) el modo en que han abordado su periodo de prácticas sin estar presencialmente en las aulas; y (c) el modo en que afrontan los cambios en la profesión.

\section{La Pérdida Del Factor Presencial En La Formación Inicial}

A partir de la cancelación de las formaciones presenciales por motivo de la COVID-19, el alumnado universitario tuvo que adaptarse para continuar adquiriendo sus aprendizajes a través de clases online. El alumnado participante en este estudio se encontraba en su periodo de prácticas en las escuelas de Educación Primaria y comenzando la realización de su Trabajo Fin de Grado cuando se declaró el fin de las clases presenciales. En este apartado se reconstruyen las impresiones de los futuros docentes en relación a cómo vivieron esta transición.

La paralización de las clases presenciales en las escuelas y las universidades hizo que las instituciones de Educación Superior tuviesen que readaptar rápidamente su formación. El alumnado de este estudio afirma que en su institución se le ofertó la posibilidad, de manera voluntaria, de continuar trabajando en la docencia online junto al maestro/tutor que les había acogido en la escuela.

Nos han ofrecido seguir trabajando de manera continuada con ellos [maestros/tutores] y me ha parecido genial. Nos han insistido que era 
opcional, pero yo quería seguir con ellos para aprender y hacer cosas útiles que les puedan ayudar. (A.)

El profesorado universitario, así como los maestros y maestras tutores, tuvieron que realizar un trabajo de reorganización de sus asignaturas hacia una forma de docencia a la que, en la mayoría de los casos, no estaban acostumbrados. Este hecho fue percibido por las y los futuros docentes, quienes consideran que el aprendizaje presencial fue sustituido, en muchas ocasiones, por un exceso en la carga de trabajo que se les ha exigido.

La tele-enseñanza conlleva algo más de trabajo que la docencia presencial, quizá debido a la falta de costumbre y por ser la novedad para aquellos que no se dedicaban a la docencia de esta manera. (S.)

Si bien la mayoría de estudiantes ha percibido la docencia online con más carga de tareas, cuando la exigencia de trabajos no ha sido muy alta el alumnado ha desarrollado una visión positiva hacia la teledocencia como una oportunidad para invertir su tiempo en otras acciones.

Con la tele-docencia me siento a gusto, posiblemente, porque en mi caso se me ha exigido lo justo como para poder trabajar a diario en diferentes tareas y no llegar a estresarme. Así, he podido sacar tiempo para el ocio y relacionarme con amigos y familia. (D.)

La universidad a la que pertenece el alumnado participante en el estudio también realizó una readaptación en la forma de evaluar. Ya no se podía hacer un seguimiento del desempeño del alumnado en las aulas. De esta forma, a las y los futuros docentes se les pidió que continuasen con la realización de unidades didácticas, evaluándose estas a través del documento escrito elaborado.

Para evaluar el Prácticum se nos exige la elaboración de tres unidades de enseñanza-aprendizaje que no vamos a poder aplicar ni vamos a poder corregir, de manera que el proceso va a quedar incompleto. (C.) 
El alumnado percibe un problema en la forma de evaluación asociada a la docencia online. Considera que es difícil poder hacer un seguimiento del proceso de aprendizaje, mediante el cual establecer unos criterios y una evaluación acorde al aprendizaje desarrollado.

\begin{abstract}
El proceso de enseñanza-aprendizaje se ve alterado ya que es más complicado establecer los criterios de evaluación para unas actividades a las que el maestro no ha podido realizar un seguimiento individualizado de cada alumno ni ha podido observar el proceso de aprendizaje. (C.)
\end{abstract}

Las y los futuros docentes consideran que a través de la docencia online no ha sido posible que el profesorado universitario y las y los maestros/tutores les hiciesen un acompañamiento individual, lo cual les ha generado dudas durante su proceso formativo que no han podido resolver en el momento.

En la parte de la tele-docencia tengo preocupaciones con ser capaz de responder de manera correcta a lo que se me pide. (A.)

Todos estos hechos han supuesto que las y los futuros docentes se hayan planteado la validez de la docencia online para la adquisición de algunas competencias docentes. Este planteamiento ha redirigido sus pensamientos hacia la forma de continuar formándose en el futuro.

Mi intención tras acabar este grado era seguir formándome en otro campo y esta situación ha hecho que me plantee la forma de abordar la siguiente formación, en el sentido de la formación a distancia o presencial. (D.)

Las competencias docentes que el alumnado considera no haber sido capaz de adquirir, debido a la pérdida del factor presencial en su formación inicial, lo reflejan, principalmente, a través de los cambios ocasionados en la asignatura del Prácticum. 
162 González-Calvo et al. - Aprender a Ser Docente Sin Estar en las Aulas

\section{El Prácticum Sin Estar En Las Aulas}

En este apartado se profundiza en la pérdida del factor presencial en la asignatura Prácticum, y las consecuencias que el alumnado considera que tendrá en su formación.

Las y los futuros docentes esperaban con una gran ilusión la realización de su periodo de prácticas, conscientes de lo importante que sería para desarrollar los aprendizajes adquiridos hasta el momento.

Veía este periodo de prácticas como una posibilidad para acercarme definitivamente a la docencia realizando una unidad de enseñanzaaprendizaje completa, con planificación, desarrollo y evaluación. (C.)

El alumnado tenía claro que el Prácticum sería una asignatura muy influyente en su desarrollo profesional. Más allá de los trabajos exigidos, las y los futuros docentes veían en este periodo de inmersión en las aulas una oportunidad para crear y fortalecer aprendizajes, a través de las relaciones sociales con otros miembros de la comunidad educativa.

El periodo de prácticas en los centros escolares trasciende más allá de las programaciones que el alumno pueda realizar y su ajuste al grupo o grupos con los que lo deba llevar a cabo (con la importancia que ello conlleva dentro del periodo). Consiste en experiencias para aprender de otros profesores, de unos alumnos con los que se establece un vínculo afectivo muy especial, con los otros maestros de prácticas, y con los padres en el caso de poder asistir a las reuniones tutoriales. (C.)

La imposibilidad de continuar con su formación en las aulas del colegio y poner en prácticas los trabajos elaborados, supone en el alumnado la percepción de que el aprendizaje que esperaban se ha visto ampliamente reducido o, incluso, se ha perdido.

Lógicamente, considero que hemos perdido una de las etapas más bonitas de toda la carrera, las últimas prácticas, compartir tanto con nuestros tutores como con nuestros compañeros el día a día, las 
experiencias, contrastar situaciones que vivimos en el centro, compartir y reflexionar sobre vivencias... (S.)

Las y los estudiantes vislumbraban su periodo de prácticas como el momento para comenzar a construir una base sólida en su identidad como docentes, lo cual ya no va a suceder. El alumnado considera que los cambios en la forma de desarrollar el Prácticum han hecho que este pierda su esencia, como una asignatura práctica en un contexto real y, por tanto, haya visto desaparecer todo su potencial formativo diferenciador.

Siento que este cuatrimestre, que prometía ser muy interesante y que me iba a permitir encontrarme con ese tipo de maestro que quiero ser, no ha supuesto los aprendizajes que yo esperaba. (C.)

El alumnado siente que la falta de inmersión en un aula durante su periodo de prácticas le supondrá un déficit formativo que no va a poder subsanar. Las y los estudiantes perciben esta experiencia perdida como irreparable en su formación.

Desde mi punto de vista, creo que esta mala suerte que hemos tenido nos deja un "vacio" no solo como estudiantes en prácticas sino también en nuestra formación académica y laboral. (D.)

De este modo, estiman que su inexperiencia en las aulas será una consecuencia que arrastrarán durante sus primeros años como docentes. $\mathrm{Su}$ falta de formación en las aulas será un aspecto que retrasará su desarrollo profesional y que deberán tratar de cubrir mediante tiempo de experiencia en la docencia u otras vías formativas.

Respecto a mi formación, considero que atrasará un poco el aprendizaje de hablar en público con más de soltura y el hecho de afrontar situaciones de dificultad, para mí, en este aspecto. En general, atrasará o cambiará el camino por el que conseguiré los aprendizajes que la experiencia de ejercer como docente presencial me hubiera aportado. (D.) 
El alumnado cree que haber perdido el factor presencial hará que se resientan sus primeras inmersiones futuras en las aulas y el trabajo con su alumnado, ya como docentes en ejercicio.

Un estado excepcional como este genera muchas consecuencias negativas, en nuestro caso, a nivel profesional, no cumplir con los objetivos previstos hace que el aprendizaje se retrase en nuestros alumnos. (G.)

Aspectos como el miedo o la inseguridad del docente novel van a verse acrecentados en el futuro. El alumnado considera que haber perdido la experiencia y el aprendizaje de su periodo de prácticas le producirá también unos sentimientos negativos durante sus primeros pasos como docentes.

Considero que para nuestra promoción, el no poder haber realizado nuestras prácticas [...] va a significar mucho más peso de sensaciones como miedo e inseguridad cuando el día de mañana comencemos a trabajar como docentes. (S.)

El estudiantado alude a la necesidad de tener que lidiar con estos nuevos sentimientos cuando sean docentes; sin embargo, su discurso muestra cómo dichos sentimientos de miedo, inseguridad e incertidumbre ya han comenzado a aparecer en ellos durante esta etapa final de su formación inicial.

\section{Aprender A Afrontar Los Cambios: Un Nuevo Reto En La Formación}

El cambio en la docencia, suscitado por el fin de la formación presencial, ha producido en el alumnado una serie de sentimientos con los que han tenido que trabajar durante esta etapa formativa. Así, en este apartado se indaga en los sentimientos desarrollados por las y los estudiantes a raíz de la actual situación de pandemia causada por la COVID-19 y los cambios sociales y formativos asociados a ella, los cuales han afectado en la evolución de sus aprendizajes.

La percepción de incertidumbre ha acompañado al alumnado en su etapa formativa durante la pandemia. Las instituciones universitarias y 
el profesorado tuvieron que realizar cambios rápidamente en sus programas docentes, con el fin de adaptar toda la docencia de forma online. Este hecho supuso que el alumnado recibiese tanta información de cómo se trabajaría, qué aspectos cambiarían o cómo se evaluaría que, en lugar de tranquilizarle, lo llenó de dudas y, con ello, de inquietudes, nerviosismo y estrés.

Al principio sentía cierta incertidumbre dado que aún nadie tenía claro cómo iban a seguir las cosas, y me estresaba recibir continuamente correos que no aclaraban nada, solo conseguían ponerme más nerviosa pensando en la acumulación de tareas que se avecinaba [...] me estreso más por lo que me imagino en mi cabeza, el miedo a que se excedan mandando trabajos o que sean demasiado complicados o improductivos. (Ca.)

Dicha inseguridad produjo en el alumnado un alejamiento hacia su proceso formativo, que se tradujo en una desmotivación hacia su aprendizaje. No saber con seguridad cómo iba a ser evaluado o las fechas para la presentación de trabajos o realización de exámenes alejaron el pensamiento de las y los estudiantes de su camino formativo, centrándolo en presuponer cómo sería el final de dicho camino.

La incertidumbre [...] hace que mi motivación varie considerablemente. El hecho de no saber con total concreción la metodología de evaluación o fechas (en el caso del TFG) hace que la duda sea aún mayor y, por tanto, la energía frente a estas tareas. (G.)

La situación de inseguridad que está viviendo el alumnado, a nivel social y formativo, les ha supuesto un desgaste anímico, que ha repercutido en su formación.

La incertidumbre que genera todo este estado excepcional hace que mis niveles de energía e implicación se vean afectados. (G.)

Las transformaciones en la docencia se dirigieron, principalmente, hacia la suplantación de las prácticas por trabajos teóricos, como analizábamos anteriormente. Este hecho condujo al alumnado a una 
situación de agobio, al tratar de dar respuesta a todas las asignaturas, que se sumaba al desgaste energético producido por la inestabilidad de la situación.

Me siento muy agobiado, no solo por la "teledocencia", sino también por el resto de quehaceres universitarios a los que tengo que responder. (A.)

El alumnado participante en el estudio fue capaz de sobreponerse a los sentimientos negativos y continuar con su aprendizaje, pero no sin acompañarlo de una sensación de nostalgia. El contacto social con otros miembros de la comunidad educativa y la experiencia en un contexto real, son factores que no se pueden aplicar de forma online, y que hacen que los estudiantes terminen su proceso formativo con un sentimiento de melancolía, producido por el contraste entre lo que esperaban de su último año de formación inicial y lo que tuvo que ser.

He realizado todas las tareas requeridas hasta el momento y he procurado participar de manera activa, pero echo de menos el contacto con el colegio a la hora de enseñar y una planificación más concreta. (A.)

Las y los futuros docentes han finalizado sus estudios con un sentimiento de tristeza, debido a la imposibilidad de completar su formación académica. Aunque han continuado trabajando de forma online con las y los maestros/tutores del colegio en la realización de actividades y unidades didácticas, al alumnado le ha quedado un sentimiento de amargura por no haber podido completar su aprendizaje en la práctica.

Me produce tristeza no poder realizar un proceso de enseñanza aprendizaje completo, ya que, con los maestros especialista del centro, estamos pudiendo diseñar actividades que se van a aplicar de manera real, aunque no en el contexto que me gustaría. (C.)

El alumnado, tras finalizar sus estudios académicos, se percibe lejos de un desarrollo profesional adecuado. No haber conseguido la 
totalidad de los aprendizajes esperados durante su último año de formación inicial deja en los futuros docentes una sensación de inconformidad respecto a su profesionalización, de cara a afrontar la docencia en una escuela.

Esta situación me deja con una sensación de inconformidad dado que no voy a poder culminar el proceso que me acerca aún más al rol profesional que el profesorado desempeña. (C.)

Finalizar su formación inicial con los sentimientos analizados, hace que el alumnado encare sus pasos futuros como docentes comenzando por revertir estos sentimientos negativos y volviendo a encontrarse con la figura del educador que querían ser.

El punto de partida para poder realizar con éxito cualquier trabajo sería encontrarnos nosotros mismos correctamente y con la actitud y energía que éste implica. (G.)

\section{Discusión}

A través del análisis narrativo de las voces del alumnado en formación inicial se ha indagado en la manera en la que experimentaron su periodo de prácticas de manera virtual como consecuencia de la actual crisis sanitaria. La profundización en sus discursos nos ha permitido comprender cómo esta situación les hace percibir que sus aprendizajes se han condicionado y cómo consideran que les afectará en su desarrollo profesional.

Los resultados muestran cómo las acciones educativas tomadas a raíz de la pandemia de la COVID-19 han puesto en riesgo la construcción de una base sólida en la identidad profesional de las y los futuros docentes. Estudios narrativos han demostrado cómo las situaciones sociales y políticas de un contexto son influyentes en la construcción de la identidad docente de las y los maestros en formación (Orellana-Fernández et al., 2020). En esta línea, la presente investigación profundiza en dicha relación a través de las narrativas de un grupo de estudiantes en formación, analizando sus perspectivas antes y después de una pandemia mundial. El estudio señala cómo antes 
de esta situación el alumnado tenía una gran esperanza depositada en su inmersión en las escuelas como docentes en prácticas, como el momento en el cual establecen una relación directa con la realidad educativa, y constituirían una base en torno a su identidad docente. Esta perspectiva concuerda con estudios anteriores que destacan la importancia de los periodos de prácticas para la construcción de la identidad (Anspal et al., 2012; Mifsud, 2018). Sin embargo, la situación de pandemia provocada por la COVID-19, que acarreó la suspensión de las clases presenciales, llevó a la reestructuración del Prácticum de forma virtual y con ello la pérdida de su carácter diferenciador y del paso del saber a la acción para que el alumnado adquiera una competencia profesional. Las y los estudiantes afirman haber perdido una experiencia potenciadora en la construcción de su identidad docente $\mathrm{y}$, en su lugar, se han visto reflejados como docentes en prácticas trabajando individualmente desde su casa, con reflexiones basadas en incertidumbres y mediante situaciones descontextualizadas. El alumnado, quien comprende que esta ha sido una situación excepcional y obligada, se aleja de aceptar este individualismo y racionalismo como la forma de construcción de su identidad y considera que no haber podido fortalecer su aprendizaje a través de la experiencia práctica, las relaciones personales y profesionales o las reflexiones contextualizadas, les ha perjudicado en su indagación por conocer y comprender la maestra y el maestro que quieren ser (LaPointe, 2010). Estos hechos parecen haber debilitado la base sobre la que las y los futuros docentes participantes en el estudio se construirán personal y profesionalmente.

El desarrollo profesional docente del alumnado en formación se ha visto frenado. Si bien el sistema educativo español no está diseñado para conseguir un verdadero desarrollo profesional en las y los docentes (Imbernón, 2017), el paso a la docencia virtual parece haberlo dificultado aún más. Los resultados muestran la insatisfacción del alumnado con las experiencias perdidas y los aprendizajes adquiridos. Si antes ya se apuntaba a la importancia de las relaciones sociales y contextuales para la formación de una identidad docente, estas son igualmente importantes en el desarrollo profesional (Hardy, 2012). Con los cambios educativos obligados por la pandemia de la COVID-19 la 
brecha entre teoría y práctica se ha ampliado. Ante la imposibilidad de haber podido desarrollar sus aprendizajes en los contextos educativos reales, el alumnado en formación ha visto eliminadas sus experiencias prácticas y las reflexiones en torno a ellas, tan importantes para su desarrollo como educadores (Körkkö et al., 2016; Poulou, 2007). Todo su aprendizaje se ha reducido al conocimiento teórico y la producción de documentos, lo cual perciben insuficiente para desarrollarse como un docente competente. De nuevo, el alumnado muestra una empatía hacia la labor de su profesorado por adaptarse rápidamente a los cambios, pero es crítico con un sistema de formación sin presencialidad, para el cuál considera que la mayoría del profesorado universitario y docentes de escuela no están preparados y que les imposibilita obtener un seguimiento adecuado y continuo de sus procesos de aprendizaje. Estos resultados aportan una nueva visión a investigaciones como las de Rodríguez et al. (2020), acerca de la perspectiva que el alumnado en formación tiene sobre la importancia y utilidad de una buena retroalimentación durante sus periodos de prácticas.

Todas las dificultades y transformaciones encontradas durante su proceso de formación han conducido al alumnado a la adquisición de una serie de sentimientos y emociones, que han sido contraproducentes en su desarrollo profesional y su identidad. El análisis de los resultados muestra que la formación del alumnado de manera virtual durante la situación de pandemia le produjo una constante incertidumbre y le deja un poso de tristeza y nostalgia, al no haber podido sentirse como un verdadero maestro en las aulas, así como de inconformidad con los aprendizajes adquiridos. La imposibilidad para que el profesorado universitario y maestras y maestros tutores acompañasen presencialmente al alumnado durante su proceso formativo le condujo a reproducir continuamente estos sentimientos, llevándolo al agotamiento anímico y la desmotivación hacia su formación. La importancia de abordar la parte más personal y emocional de un maestro en formación durante sus prácticas ha sido ya estudiada (Bloomfield, 2010; Yuan \& Lee, 2016), sin embargo el presente estudio plantea la dificultad de conseguirlo y sus consecuencias negativas cuando no existe un contacto continuo y cercano entre mentores y 
alumnado. De esta forma, los resultados muestran cómo el alumnado se percibe a sí mismo con una fuerte carga emocional para abordar los primeros años de docencia, así como con un aprendizaje y experiencia menor al esperado.

El alumnado prevé que en sus primeros años como docentes deberán recuperar la experiencia perdida durante su formación inicial y los aprendizajes asociados. Sin embargo, este pensamiento podría ser perjudicial para ellos ya que, como la investigación científica ya ha demostrado, en los primeros años de docencia existe un fuerte contraste entre lo deseado y las posibilidades reales de actuación en un docente novel (Pillen et al., 2013), situación que se acrecentará en un docente principiante con una nula experiencia previa. A este hecho hay que sumarle la perspectiva que el propio alumnado muestra sobre las sensaciones de inseguridad o los miedos que van a arrastrar en sus primeras inmersiones en el aula debido a su falta de práctica y el trabajo reflexivo en torno a ella, lo cual incrementará, de nuevo, los propios sentimientos negativos que ya suelen aflorar en un maestro novel (De Neve \& Devos, 2017; Fenwick, 2011). En este sentido, el análisis de los datos nos dirige a tener en cuenta todas estas problemáticas cuando el actual alumnado en formación se incorpore a las aulas y hacerlos desde métodos formativos presenciales, que aseguren al futuro docente la posibilidad de colaborar con docentes en ejercicio y reflexionar colectivamente en los contextos, como la investigación-acción participativa (Barba-Martín, 2019; Barba-Martín et al., 2018).

\section{Conclusiones}

En este trabajo se han plasmado las experiencias, temores y expectativas que las y los futuros docentes tienen en torno a la enseñanza en un futuro a corto plazo en un contexto y un momento social y económico críticos generados por la COVID-19. Los resultados del estudio muestran que el alumnado percibe el Prácticum como la asignatura más influyente en su desarrollo profesional y que, debido a la pérdida del factor presencial en las aulas escolares durante estos meses de crisis sanitaria, puede tener lugar un déficit formativo cuyas consecuencias arrastrarán durante sus primeros años como docentes. A 
pesar de que el alumnado se ha (re)adaptado con la mayor celeridad posible a un formato virtual de las clases, los cambios sufridos en el plano educativo produjeron que brotaran en el alumnado diversas emociones, como han sido la incertidumbre ante los nuevos procesos de aprendizaje, la melancolía por una formación presencial, la desmotivación en los aprendizajes, la tristeza por sentir que no pueden completar su formación académica, y otras.

Esta situación emergente también ha mostrado que lo presencial es imprescindible a la hora de entender procesos educativos que afecten al alumnado y al profesorado, sin olvidar que la educación emocional y corporal no pueden quedar ajenas al desarrollo profesional docente; debiendo considerar para el futuro las bondades y limitaciones de lo virtual, y con ello ofrecer un enfoque crítico que desnaturalice la neutralidad del mundo tecnológico. La suspensión de las actividades docentes presenciales para dar paso a la virtualización ha supuesto que profesorado y alumnado tengan una elevada presión tanto en su trabajo como en intentar adaptarse al nuevo escenario. También es necesario argumentar que es difícil pensar que hacer una transición a una docencia online es cosa de unos pocos días, principalmente porque se requieren procesos largos de formación, de (re)diseño de las asignaturas y de infraestructuras que soporten la demanda. Asimismo, resulta complicado salir de esta vorágine en la que pocas y pocos, principalmente las y los profesionales que trabajan dentro de ella, defienden una educación de calidad, y una mayoría que se manifiesta en contra.

La investigación desarrollada pone de manifiesto que, a raíz de este proceso de adaptación urgente a la virtualidad, el alumnado se ha encontrado en un sendero en el que la inseguridad, la incertidumbre y otros sentimientos han aflorado al haber perdido una experiencia potenciadora en la construcción de su identidad docente, como es el Prácticum.

Si bien la intención de nuestro estudio, como de cualquier investigación cualitativa, no es hacer generalizaciones empíricas acerca de la influencia que la crisis sanitaria derivada de la COVID-19 tiene sobre la identidad profesional de las y los futuros docentes, la muestra 
172 González-Calvo et al. - Aprender a Ser Docente Sin Estar en las Aulas

de participantes con la que hemos contado podría considerarse una limitación de la investigación.

Como líneas de investigación futuras pretendemos seguir indagando en los procesos de construcción de la identidad docente que surjan a raíz de las consecuencias de la crisis sanitaria, abogando por una formación inicial que permita comprender las experiencias y vivencias de las y los futuros maestros como ejes sobre los que giran gran parte de su práctica y convicciones pedagógicas.

\section{Referencias}

Anspal, T., Eisenschmidt, E., \& Löfström, E. (2012). Finding myself as a teacher: Exploring the shaping of teacher identities through student teachers narratives. Teachers and Teaching: Theory and Practice, $18(2)$

197-216. https://doi.org/10.1080/13540602.2012.632268

Atkinson, D. (2004). Theorising how student teachers form their identities in initial teacher education. British Educational Research Journal, 30(3), 379-394. https://doi.org/10.1080/01411920410001689698

Barba-Martín, R. A. (2019). La investigación-acción participativa desde la mirada de las maestras participantes en un proceso de formación permanente del profesorado sobre educación inclusiva. Tesis doctoral. Segovia: Universidad de Valladolid.

Barba-Martín, R. A., Sonlleva-Velasco, M., \& García-Martín, N. (2018). "Presencia, participación y progreso": el aprendizaje basado en proyectos en la trayectoria de una maestra en formación. Revista Electrónica Interuniversitaria de Formación del Profesorado, 21(2), 13-25. http://dx.doi.org/10.6018/reifop.21.2.323201

Bloomfield, D. (2010). Emotions and 'getting by': a pre-service teacher navigating professional experience. Asia-Pacific Journal of Teacher Education, 38(3), 221-234. https://doi.org/10.1080/1359866X.2010.494005

Bolívar, A., \& Domingo, J. (2019). La investigación (auto)biográfica en Educación. Octaedro. 
Britzman, D. P. (2007). Teacher education as uneven development: toward a psychology of uncertainty. International Journal of Leadership in Education, 10(1), 1-12.

Clandinin, D. J. (2007). Handbook of narrative inquiry. Mapping a methodology.

Sage

Publications.

https://dx.doi.org/10.4135/9781452226552

Dale, G. A. (2000). Distractions and coping strategies of elite decathletes during their memorable performances. The Sport Psychologist, 14, 17-41. https://doi.org/10.1123/tsp.14.1.17

Day, C., Elliot, B., \& Kington, A. (2005). Reform, standards and teacher identity: challenges of sustaining commitment. Teaching and Teacher Education, 21(5), 563-577. https://doi.org/10.1016/j.tate.2005.03.001

De Neve, D., \& Devos, G. (2017). Psychological states and working conditions buffer beginning teachers' intention to leave the job. European Journal of Teacher Education, 40, 6-27. http://dx.doi.org/10.1080/02619768.2016.1246530

Diez-Gutiérrez, E. \& Gajardo-Espinoza, K. (2020). Educar y Evaluar en Tiempos de Coronavirus: la Situación en España. Multidisciplinary Journal of Educational Research, 10(2), 102134. https://doi.org/10.4471/remie.2020.5604

Fenwick, A. (2011). The first three years: experiences of early career teachers. Teachers and Teaching, 17(3), 325-343. https://doi.org/10.1080/13540602.2011.554707

García-Peñalvo, F. J., Corell, A., Abella-García, V., \& Grande, M. (2020). La evaluación online en la Educación Superior en tiempos de la COVID-19. Education in the Knowledge Society, 21. https://doi.org/10.14201/eks.23013

González-Calvo, G., \& Arias-Carballal, M. (2017). A teacher's personal-emotional identity and its reflection upon the development of hisprofessional identity. The Qualitative Report, 22(6), 1693-1709.

González-Calvo, G., \& Fernández-Balboa, J. M. (2018). A qualitative analysis of the factors determining the quality of relations between a novice physical education teacher and his students' families: implications for the development of professional 
174 González-Calvo et al. - Aprender a Ser Docente Sin Estar en las Aulas

identity. Sport, Education and Society, 23(5), 491-504. https://doi.org/10.1080/13573322.2016.1208164

Graham, A., \& Phelps, R. (2003). 'Being a teacher': developing teacher identity and enhancing practice through metacognitive and reflective learning processes. The Australian Journal of Teacher Education, $27(2)$,

11-24. http://dx.doi.org/10.14221/ajte.2002v27n2.2

Gubrium, J. F., \& Holstein, J. A. (2009). Analyzing narrative reality. Sage.

Hardy, I. (2012). The politics of teacher professional development: policy, research and practice. Routledge.

Hong, J. Y. (2010). Pre-service and beginning teachers' professional identity and its relation to dropping out of the profession. Teaching and Teacher Education, 26(8), 1530-1543. https://doi.org/http://dx.doi.org/10.1016/j.tate.2010.06.003

Imbernón, F. (2007). La formación y el desarrollo profesional del profesorado. Hacia una nueva cultura profesional. Graó.

Imbernón, F. (2017). Ser docente en una sociedad compleja: la dificil tarea de enseñar. Graó.

Korthagen, F. (2004). In search of a the essence of a good teacher: Towards a more holistic approach in teacher education. Teaching and Teacher Education, 20, 77-97. https://doi.org/10.1016/j.tate.2003.10.002

Korthagen, F. (2010). La práctica, la teoría y la persona en la formación del profesorado. Revista Interuniversitaria de Formación del Profesorado, 68(24,2), 83-101.

Körkkö, M., Kyrö-Ämmälä, O., \& Turunen, T. (2016). Professional development through reflection in teacher education. Teaching and Teacher Education, 55, 198-206. https://doi.org/10.1016/j.tate.2016.01.014

LaPointe, K. (2010). Narrating career, positioning identity: career identity as a narrative practice. Journal of Vocational Behavior, 77(1), 1-9. https://doi.org/10.1016/j.jvb.2010.04.003

Le Baron, C. (2006). Microethnography. In V. Jupp (Ed.), The Sage Dictionary of Social Research Methods, (pp. 177-179). Sage Publications. https://dx.doi.org/10.4135/9780857020116.n119 
International and Multidisciplinary Journal of Social Sciences, 9(2) 175

Libarkin, J. C., \& Kurdziel, J. (2002). Research methodologies in Science Education: qualitative data. Journal of Geoscience Education, $\quad 50$, 195-200.

https://doi.org/10.1080/10899995.2002.12028052

Lincoln, Y. S., \& Guba, E. (1985). Naturalistic inquiry. Sage.

Llorens-Largo, F., \& Fernández, A. (2020). Coronavirus, la prueba del algodón de la universidad digital. https://bit.ly/2Rm917X

Lopes, A. (2009). Teachers as professionals and teachers' identity construction as an ecological construct: an agenda for research and training drawing upon a biographical research process. European Educational Research Journal, 8(3), 461-475. http://www.wwwords.co.uk/rss/abstract.asp? ${ }^{\prime}=e e r j \& a i d=3705 \&$ doi $=1$

Loughran, J. (2005). Researching teaching about teaching: self-study of teacher education practices. Studying Teacher Education, 1(1), 516. https://doi.org/10.1080/17425960500039777

Mifsud, D. (2018). Professional identities in initial teacher education: the narratives and questions of teacher agency. Springer.

Miles, M. B., \& Huberman, A. (1994). Data management and analysis methods. In N. K. Denzin \& Y. S. Lincoln (Eds.), Handbook of Qualitative Research (pp. 428-444). Sage.

Orellana-Fernández, R., Merellano-Navarro, E., Berríos-Villarroel, A., \& Almonacid-Fierro, A. (2020). Teachers in dictatorship and democracy: biographical perspective of teachers at the end of a professional career. Revista Brasileira de Educacao, 25. https://doi.org/10.1590/s1413-24782019250007

Orland-Barak, L., \& Maskit, D. (2011). Novices 'in story': what firstyear teachers' narratives reveal about the shady corners of teaching. Teachers and Teaching, 17(4), 435-450. https://doi.org/10.1080/13540602.2011.580520

Pence, H., \& Macgillivray, I. (2008). The Impact of an International Field Experience on Preservice Teachers. Teaching and Teacher Education: An International Journal of Research and Studies, 24(1), 14-25. http://dx.doi.org/10.1016/j.tate.2007.01.003 
176 González-Calvo et al. - Aprender a Ser Docente Sin Estar en las Aulas

Perrenoud, P. (2010). La formación del profesorado: un compromiso entre visiones inconciliables de la coherencia. Revista Interuniversitaria de Formación del Profesorado, 68, 103-122.

Pillen, M., Beijaard, D., \& den Brok, P. (2013). Tensions in beginning teachers' professional identity development, accompanying feelings and coping strategies. European Journal of Teacher Education, 36(3), 240-260. https://doi.org/10.1080/02619768.2012.696192

Poulou, M. (2007). Student-teachers' concerns about teaching practice. European Journal of Teacher Education, 30(1), 91-110. https://doi.org/10.1080/02619760600944993

Rodgers, C., \& Scott, K. (2008). The development of the personal self and professional identity in learning to teach. In M. CochranSmith, S. Feiman-Nemser, D. J. McIntyre, \& K. E. Demers (Eds.), Handbook of research on teacher education: Enduring questions and changing contexts (pp. 732-755). Routledge.

Rodríguez, J., McKinney, T., Powell, S., Walker, Z., \& Vince Garland, K. (2020). Was this feedback useful? Examining the observation and feedback process for pre-service teachers. Teaching Education, 31(2), 144-161. https://doi.org/10.1080/10476210.2018.1508281

Ruohotie-Lyhty, M. (2013). Struggling for a professional identity: two newly qualified language teachers' identity narratives during the first years at work. Teaching and Teacher Education, 30(0), 120129. https://doi.org/http://dx.doi.org/10.1016/j.tate.2012.11.002

Smith, B. (2010). Narrative inquiry: ongoing conversations and questions for sport and exercise psychology research. International Review of Sport and Exercise Psychology, 3, 87107. https://doi.org/10.1080/17509840903390937

Søreide, G. E. (2006). Narrative construction of teacher identity: Positioning and negotiation. Teachers and Teaching: Theory and Practice, $12(5)$, 527-547. https://doi.org/10.1080/13540600600832247

Sparkes, A., \& Smith, B. (2014). Qualitative research methods in sport, exercise and health: from process to product. Routledge. https://doi.org/10.4324/9780203852187 
Trujillo, F., Fernández, M., Segura, A., \& Jiménez, M. (2020). Escenarios de evaluación en el contexto de la pandemia por la covid-19: la opinión del profesorado. SantillanaLab.

Watson, C. (2007). Narratives of practice and the construction of identity in teaching. Teachers and Teaching: Theory and Practice, 12(5), 509-526. https://doi.org/10.1080/13540600600832213

Yuan, R., \& Lee, I. (2016). 'I need to be strong and competent': a narrative inquiry of a student-teacher's emotions and identities in teaching practicum. Teachers and Teaching: Theory and Practice, https://doi.org/10.1080/13540602.2016.1185819

Gustavo González-Calvo is partial time lecturer at the Departamento de Didáctica de la Expresión Musical, Plástica y Corporal at the University of Valladolid

Raúl A. Barba-Martín is postdoctorate researcher at the Departamento de Didáctica de la Expresión Musical, Plástica y Corporal at the Univeristy of Valladolid

Daniel Bores-García is partial time lecturer at the Department of Fisioterapia, Terapia Ocupacional, Rehabilitación y Medicina Fisica at the University Rey Juan Carlos

Vanesa Gallego-Lema is postdoctorate researcher at the research group Elkarrikertuz at the University of the Basque Country

Contact adress: raulalberto.barba@uva.es 